\title{
A preservação e a disseminação da história e cultura africana e afro-brasileira a partir do colecionismo nas unidades de informação no Brasil
}

\author{
Graziela dos Santos Lima \\ Universidade Estadual Paulista, Faculdade de Filosofia e Ciências \\ Departamento de Ciência da Informação, Marília, SP, Brasil \\ graziela.lima@unesp.br \\ Cláudia Pereira de Jesus Carvalho \\ Universidade Estadual Paulista, Faculdade de Filosofia e Ciências \\ Departamento de Ciência da Informação, Marília, SP, Brasil \\ claudia.pjc@yahoo.com \\ Carlos Cândido de Almeida \\ Universidade Estadual Paulista, Faculdade de Filosofia e Ciências \\ Departamento de Ciência da Informação, Marília, SP, Brasil \\ carlos.c.almeida@unesp.br
}

DOI: https://doi.org/10.26512/rici.v13.n1.2020.29498

Recebido/Recibido/Received: 2020-01-02

Aceitado/Aceptado/Accepted: 2020-02-04

Resumo: Esta pesquisa tem como objetivo geral identificar a presença das coleções (de objetos e bibliográficas) que representem a história dos negros e as tradições culturais africanas no Brasil. Os objetivos específicos consistem em discorrer sobre o colecionismo; discutir a preservação da memória e cultura africana e afro-brasileira, além de destacar as principais unidades de informação com coleções que representam a cultura africana e afro-brasileira. Considera-se que a disseminação da memória africana e afro-brasileira é potencializada por essas coleções, pois colecionar artefatos e documentos que retratam a história e a cultura negra representa um ato de resistência e evidência das práticas culturais das populações negras. Tem uma abordagem teórico-bibliográfica, com propósito crítico e reflexivo pautado nas teorias do colecionismo e da memória. Foram identificadas dentro do contexto brasileiro por meio de mapeamentos as principais unidades de informações especializadas, a preservação em diversas tipologias de coleções que contribuem para conservação e fortalecimento da memória, história, cultura e identidade dos africanos e afro-brasileiros. Estas unidades colecionadoras podem ser assim classificadas: Centros de Estudos Africanos (6 instituições), Núcleos de Estudos Afrobrasileiros (139 instituições) e Museus afros (36 instituições), entre organizações públicas e privadas. Essas coleções são formadas por objetos informacionais que identificam períodos históricos, registrando um passado, o qual é recordado por meio da memória, intensificada pelos objetos, cujos significados, via representações, se fazem presentes na mente do indivíduo e dão sentido à experiência. Atreladas às informações orais, as coleções representam valiosas fontes de informação e conhecimento. Portanto, os registros como relatos, inventários, objetos museológicos funcionam como informações primárias para desvendar e representar histórias e culturas passadas preservando a memória das populações de origem africana no Brasil. Para as futuras pesquisas é importante selecionar um museu privado para identificação dos objetos, reconstituição de sua origem e os significados tanto para o museu quanto para a comunidade ao seu redor.

Palavras-chave: memória. colecionismo. cultura afro-brasileira. identidade afro-brasileira. Ciência da informação. 


\section{The preservation and dissemination of African and Afro-Brazilian history and culture based on collecting in information units in Brazil}

Abstract This research aims to identify the presence of collections (of objects and bibliographic) that represent the history of blacks and African cultural traditions in Brazil. The specific objectives are to talk about collecting; discuss the preservation of African and Afro-Brazilian memory and culture and highlight the main information units with collections representing African and Afro-Brazilian culture. The dissemination of African and Afro-Brazilian memory is potentiated by these collections, since collecting artifacts and documents that portray black history and culture represents an act of resistance and evidence of the cultural practices of black populations. It has a theoretical and bibliographical approach, with critical and reflexive purpose based on the theories of collecting and memory. Within the Brazilian context, the main units of specialized information, the preservation in various types of collections that contribute to the conservation and strengthening of the memory, history, culture and identity of Africans and Afro-Brazilians were identified through mappings. These collector units can be classified as follows: African Study Centers (6 institutions), Afro-Brazilian Study Centers (139 institutions) and Afros Museums (36 institutions), between public and private organizations. These collections are formed by informational objects that identify historical periods, recording a past, which is remembered through memory, intensified by objects, whose meanings, through representations, are present in the individual's mind and give meaning to the experience. Linked to oral information, collections represent valuable sources of information and knowledge. Therefore, records such as reports, inventories, museum objects function as primary information to unravel and represent past histories and cultures preserving the memory of populations of African origin in Brazil. For future research it is important to select a private museum to identify objects, reconstitute their origins and meanings for both the museum and the surrounding community.

Keywords: memory. collecting. Afro-Brazilian culture. Afro-Brazilian identity. Information Science.

\section{La preservación y difusión de la historia y cultura africana y afrobrasileña basada en la colección de información en las unidades de Brasil.}

Resumen: El objetivo general de la investigación es identificar la presencia de colecciones (de objetos y bibliografías) que representan la historia de los negros y las tradiciones culturales africanas en Brasil. Los objetivos específicos son hablar sobre coleccionar; discuta la preservación de la memoria y la cultura africana y afrobrasileña, además de destacar las principales unidades de información con colecciones que representan la cultura africana y afrobrasileña. Se considera que la difusión de la memoria africana y afrobrasileña se ve reforzada por estas colecciones, ya que la recopilación de artefactos y documentos que retratan la historia y la cultura negras representa un acto de resistencia y evidencia de las prácticas culturales de las poblaciones negras. Tiene un enfoque teórico-bibliográfico, con un propósito crítico y reflexivo basado en las teorías del coleccionismo y la memoria. Las principales unidades de información especializada se identificaron dentro del contexto brasileño mediante el mapeo, la preservación en diferentes tipos de colecciones que contribuyen a la conservación y el fortalecimiento de la memoria, historia, cultura e identidad de africanos y afrobrasileños. Estas unidades de recolección se pueden clasificar de la siguiente manera: Centros de Estudios Africanos (6 instituciones), Centros de Estudios Afrobrasileños (139 instituciones) y Museos Africanos (36 instituciones), entre organizaciones públicas y privadas. Estas colecciones están formadas por objetos informativos que identifican períodos históricos, registrando un pasado, que se recuerda a través de la memoria, intensificado por objetos, cuyos significados, a través de representaciones, están presentes en la mente del individuo y le dan sentido a la experiencia. Vinculadas a la información oral, las colecciones representan valiosas fuentes de información y conocimiento. Entonces, los registros como informes, inventarios, objetos de museo funcionan como información primaria para desentrañar y representar historias y culturas pasadas al tiempo que preservan la memoria de las poblaciones de origen africano en Brasil. Para futuras investigaciones, es importante seleccionar un museo privado para identificar los objetos, reconstruir su origen y significado tanto para el museo como para la comunidad que lo rodea.

Palavras-chaves: memoria. coleccionar. cultura afrobrasileña. identidad afrobrasileña. Ciencia de la información. 


\section{Introdução}

As práticas colecionistas existem desde os primórdios da humanidade, sendo resultado do desejo de selecionar e guardar coisas. As coleções relacionam-se ainda com outras características naturais da humanidade, como classificar, nomear, atribuir significados, funções às coisas, pessoas e lugares.

A pesquisa tem como objetivo geral identificar a presença das coleções (de objetos e bibliografias) que representem a história da população negra e as tradições culturais africanas no Brasil. Os objetivos específicos consistem em discorrer sobre o colecionismo; discutir a preservação da memória e cultura africana e afro-brasileira, além de destacar as principais unidades de informação com coleções que representam a cultura africana e afro-brasileira.

Considera-se que a disseminação da memória africana e afro-brasileira é potencializada por essas coleções, pois colecionar artefatos e documentos que retratam a história e a cultura negra representa um ato de resistência e evidência das práticas culturais das populações negras. O texto possui uma abordagem teórico-bibliográfica, com propósito crítico e reflexivo pautado nas teorias do colecionismo e da memória relacionada à história e cultura africana e afro-brasileira.

Isto posto, as coleções de uma maneira geral são formadas por objetos informacionais que identificam períodos históricos, registrando um passado, o qual é recordado por meio da memória, intensificada pelos objetos, cujos significados, via representações, se fazem presentes na mente do indivíduo e dão sentido à experiência. Atreladas às informações orais, as coleções representam valiosas fontes de informação e conhecimento. Portanto, consideramos importante e necessário detectar e discutir a presença (ou ausência) de coleções (tanto de objetos quanto bibliográficas) que representem a história dos negros no país, as tradições culturais africanas e sobre a importância da preservação e disseminação dessas coleções.

\section{Colecionismo}

O colecionismo surge de uma característica natural do ser humano que é classificar, nomear, atribuir significados, funções às coisas, pessoas e lugares. Em sua etimologia, a palavra coleção deriva do latim collectio + onis, que é compilação, coletânea, reunião (BUENO, 1974). Entretanto, a despeito do que elementarmente possa parecer, coleções não são apenas o mero acúmulo de objetos, como pode ser visto pelo senso comum, é possível análises mais profundas sob diferentes perspectivas, como social, cultural, psicológica, econômica, semântica. $O$ universo semântico contido numa coleção pode ser muito rico, pois para o colecionador "a coleção não é um mero amontoado de objetos iguais, mas cada um 
conta uma história e possui um significado próprio por si" (OLIVEIRA; HOLANDA; MACIEL, 2016, p. 36).

O geólogo Menegat $(2005$, p. 5) defende que a sobrevivência e evolução humana está relacionada ao colecionismo, afirmando que "poucas atividades cognitivas humanas têm a transversalidade e duração do colecionismo". No entanto, o ato de selecionar coisas e estabelecer significados para elas tem origem desde a pré-história e é um processo que constitui a evolução da espécie humana.

Coletar, selecionar, definir diferenças e semelhanças, agrupar, traçar tentativas de explicação, são coisas que todas as pessoas fazem, ainda que sem consciência do fato, "todos seres humanos de alguma forma colecionam coisas, afetos e memórias" (MENEGAT, 2005, p. 6). Algumas coleções servem para explicar o passado, pois são uma reconstituição de parte dele, nos apresentam fragmentos de como eram as coisas, lugares, animais, plantas. Estudando as coleções de um dado momento histórico, é possível entender melhor certos fenômenos.

O Dicionário brasileiro de terminologia arquivística (ARQUIVO NACIONAL, 2005) estabelece coleção como conjunto de documentos com características comuns, reunidos intencionalmente e colecionador como entidade coletiva, pessoa ou família responsável pela formação de uma coleção. Definições essas que coadunam com o conceito de Homulos (1990) que vê bibliotecas, arquivos e museus como instituições colecionadoras.

Pomian (1984, p. 53) traça um limite bem peculiar de coleção, citado na grande maioria dos estudos sobre colecionismo; o historiador e filósofo entende coleção como "qualquer conjunto de objetos naturais ou artificiais, mantidos temporária ou definitivamente fora do circuito das atividades econômicas, sujeitos a uma proteção especial num local fechado preparado para esse fim, e expostos ao público". Esses locais, no âmbito institucional, costumam ser museus, arquivos e bibliotecas, possuindo cada um suas coleções características, apesar de apresentarem diversos pontos e objetos em comum. Assim, essas instituições teriam o que Pomian denomina de "coleção de coleções", reunindo diversos tipos de coleções em um mesmo espaço.

Valis (2013, p. 561) argumenta que "Os objetos são parte do que nos confere humanidade. Um mundo sem objetos é um mundo sem seres humanos". Assim, a humanidade foi, ao longo do tempo, cercando-se de objetos, precisamos deles para nos relacionar com o mundo e com as outras pessoas, eles são muitas vezes mediadores, nos ajudam, nos protegem e nos completam.

Baudrillard (2004) define duas possibilidades de classificação de um objeto: como instrumento e como signo, e embora essas duas funções sejam antagônicas, não são 
mutuamente excludentes. Assim, existem duas incumbências para o objeto: ser utilizado e ser possuído, ou seja, um papel material e outro simbólico. O objeto funcional denota uma categoria social, pode relacionar-se a qualquer indivíduo. $\mathrm{O}$ objeto simbólico denota uma categoria privativa, relaciona-se a somente uma pessoa. E somente o último pode se tornar objeto de coleção.

Renault (2015, p. 13) afirma que "a formação de coleções na sociedade reúne um amplo aspecto de coisas e contextos que vão desde a curiosidade científica até aspectos estéticos, religiosos e até mesmo funcionais.".

Para Fonseca (2005, p. 183) colecionar “[...] é, em última análise, uma tentativa de, em termos inconscientes, re-tomar, re-utilizar, re-viver, re-ver, re-memorar, ressignificar e mesmo re-criar épocas, objetos e os afetos a eles ligados". A humanidade imputou funções e significados às coisas físicas com as quais se deparou ao longo do tempo, algumas encontradas na própria natureza, outras criados pelos próprios homens.

Azevedo (2015, p. 18) declara que as coleções são uma forma de recuperar o passado: "A guarda dos objetos remete a fatos e acontecimentos relevantes para a sociedade, por exemplo, a guarda de despojos de guerra, ou mesmo a guarda de fotografias de família, e objetos que retomem sentimentos vividos anteriormente". Assim sendo, com a coleção têmse uma possibilidade de reviver, ainda que de forma simbólica, episódios que marcaram a vida de uma pessoa ou de uma coletividade. Portanto, ao passo que coleções pessoais ecoam lembranças individuais, coleções institucionais ecoam a memória coletiva.

\section{Memória e cultura africana e afro-brasileira}

Os processos de formação de coleções se vinculam ao desejo de preservar memórias, que são lembranças/recordações de algo ocorrido no passado e que são expressa de forma fragmentada ou não no presente, de levar ao futuro pedaços representativos que permitirão reconstituir um contexto a partir de diferentes perspectivas. Dentro de um mesmo período histórico, diferentes coleções compostas por objetos semelhantes, contarão diferentes narrativas, pois foram construídas por pessoas ou instituições com propósitos e perspectivas diferentes.

Pedrão e Bizello (2016) argumentam que a história e a memória de uma sociedade podem ser reconstituídas através das coleções . Nesse sentido, a relação coleção/colecionador é permeada por fatores sentimentais e memoriais, completamente pessoais e subjetivos, porém, há a possibilidade das coleções comportarem também memória social, com valor representativo não só para o colecionador, mas para a sociedade; conforme apontam Pedrão e Bizello (2016, p. 830), a memória de uma coleção "pode se tratar de uma memória histórica e 
cultural, não se limitando apenas ao colecionador. Ela pode se estender a uma população local ou até maior dependendo das proporções da coleção e de quanto ela é divulgada". Por essas e outras razões, muitas coleções particulares acabam sendo incorporadas à unidades informacionais como bibliotecas, arquivos e museus.

Muitas das memórias que persistiram ao longo do tempo sobre a história e cultura africana e afro-brasileira é resultado de fontes primárias armazenadas em arquivos, cúrias metropolitanas, museus, centros e núcleos de estudos africanos e afro-brasileiros que registram e atestam que as populações de origem africana foram participantes ativos no desenvolvimento do Brasil. Além dos registros, tais como documentos relacionados a processos criminais e de liberdade, censos, relatórios de chefes de polícia, inventários, registros paroquiais, cartas de alforrias, cartas de viajantes, objetos fósseis, objetos cerâmicos entre outros, bem como relatos orais serviram para reconstrução da história e memória dos africanos e afro-brasileiros que deixaram vestígios na sociedade brasileira.

Muitas das histórias contadas sobre os africanos e seus descendentes de forma fragmentada para atingir um esquecimento, ou apagamento histórico resultam de um investimento político em torno do branqueamento da nação de modo a retirar as "marcas da presença africana" (CARDOSO, 2008, p. 20) na sociedade brasileira. Essas histórias, moldadas por um pensamento europeu oriundo da modernidade, baseada na ordem linguística de ver e pensar o mundo, possibilitou em criação de uma história na qual sub-representa a população negra. Essa ordem linguística também influenciou na organização dos objetos informacionais nas unidades de informação, em especial, as que tratam da temática citada.

Segundo Cardoso (2008) existiu na década de 1990 dois movimentos intelectuais que por meio de suas obras baseada em críticas do mundo ocidental que possibilita repensar práticas do conhecimento humanístico. O primeiro, formado por intelectuais que por meio das lutas anti-coloniais nos países periféricos, bem como, das minorias políticas marginalizadas nos países centrais, procuraram repensar "maneiras pela qual a cultura ocidental buscou apreender a diversidade humana e, ao mesmo tempo, foi se instituindo como referencial absoluto para pensar as 'outras' culturas" (CARDOSO, 2008, p. 24). Esses pesquisadores que evidenciam outras culturas são pesquisadores teóricos dos pós-colonial, decolonial e estudos subalternos tais como Stuart Hall (1997), Homi Bhabha (1999), Edward Said (2007), Achille Mbembe (2018), Grada Kilomba (2019), Gayatri Chakravorty Spivak (2010), Maria Paula Meneses (2010), Anibal Quijano (2010), Ramon Grosfoguel (2005), Boaventura de Souza Santos (2010), Nelson Maldonato-Torres (2005), entre outros, que sinalizam a ordem discursiva construidas no século XIX. Nesse sentido, esses pesquisadores têm desconstruídos narrativas colonizadas e evidenciando outros vieses, a partir do sul global. 
O segundo movimento de intelectuais surgiu de movimentos políticos, como o movimento negro. Muitos historiadores e muitas historiadoras sociais revisitaram velhos temas reconstruíram a história e cultura africana e afro-brasileira "produziram documentos que tornaram possível, não apenas incluir na historiografia brasileira a perspectiva dos excluídos, como distanciar a produção histórica dos projetos institucionais das elites brasileiras" (CARDOSO, 2008, p. 25) tendo um olhar sociocultural.

As unidades de informação possuem coleções de diversos objetos que estão baseadas em uma ordem discursiva. A forma de organizar os objetos que dá vazão a presença e ausência legitimam certas narrativas. Ultimamente, nas unidades de informações em instituições públicas tem-se verificado uma organização colonizada da memória e histórias da população negra, o que Ogbechie (2014) chama de unidades de informações ${ }^{1}$ ocidentais.

As unidades de informações ocidentais de origem colonialista, universaliza os objetos na concepção ocidental e marginaliza os modos de colecionar de outras culturas (OGBECHIE, 2014). Baseado em Foucault, Ogbechie (2014) relata que o modo de classificar insinua a relação entre as unidades de informação e os objetos. E também a forma como é organizado afeta e modela a maneira que o público vê e consome as informações. Portanto, a organização via classificação de objetos em unidade de informação é uma prática discursiva (FOUCAULT, 1973), pois evidencia um determinado discurso embutidos nas práticas de representar e organizar as coleções.

O discurso dos objetos, ou as narrativas possíveis de serem materializadas ou representadas são fragmentos da memória (MENDES, 2014), ou seja, memória seletiva e quando esta participa de uma coleção, o colecionador tem o poder de selecionar o que quer ou não lembrar a partir desses objetos. Dessa forma, as coleções de materiais, ou objetos que remetem à memória e à história das populações de origem africanas no país é uma forma de estabelecer sua importância, testemunho do tempo e da história e acontecimento e ao mesmo tempo a sua invisibilidade, dependendo da instituição que a organiza.

\section{Coleções afro-brasileiras em unidades informacionais}

Após comentar os conceitos de coleções, memória, representação e cultura africana, nesta seção será apresentado um levantamento sobre a presença de coleções afro-brasileiras detectadas em Centros de Estudos Africanos, Núcleos de Estudos Afro-brasileiros e Museus, considerando unidades físicas e digitais.

\footnotetext{
${ }^{1} \mathrm{~A}$ autora, em sua pesquisa, não utiliza o termo unidades informacionais, pois a pesquisa dela se refere a uma das unidades informacionais, o museu.
} 
Foram identificadas por meio de mapeamentos as principais unidades de informações especializadas, dentro do contexto brasileiro que preservam por meio de diversas tipologias de coleções e contribuem para conservação e fortalecimento da memória, história, cultura e identidade dos africanos e afro-brasileiros, como segue: Centros de Estudos Africanos: 6, Núcleos de Estudos Afro-brasileiros: 139 e Museus afros: 36, entre instituições públicas e privadas, conforme os quadros abaixo.

Quadro 1. Centros de Estudos Africanos

\begin{tabular}{|l|l|l|}
\hline \multicolumn{1}{|c|}{ Centro de Estudos Africanos } & \multicolumn{1}{c|}{ Instituições } & \multicolumn{1}{c|}{ Região } \\
\hline Centro de Estudos Afro-Orientais (CEAO) & Universidade Federal da Bahia & Nordeste \\
\hline Centro de Estudos Afro-Asiáticos (CEAA) & Universidade Cândido Mendes (UCAM) & Sudeste \\
\hline Centro de Estudos Africanos no Brasil (CEA) & $\begin{array}{l}\text { Faculdade de Filosofia, Letras e Ciências } \\
\text { Humanas da Universidade de São Paulo } \\
\text { (FFLCH/USP). }\end{array}$ & Sudeste \\
\hline Centro de Estudos Africanos no Brasil & UFMG & Sudeste \\
\hline Laboratório de Estudos Africanos (LeÁfrica) & IFCS-UFRJ & Sudeste \\
\hline Centro Brasileiro de Estudos Africanos (CEBRAFICA) & $\begin{array}{l}\text { Universidade Federal do Rio Grande do Sul } \\
\text { (UFRGS) }\end{array}$ & Sul \\
\hline
\end{tabular}

Fonte: elaborado pelos autores

Quadro 2. NEABs

\begin{tabular}{|c|c|c|}
\hline NEABS e núcleos correlatos & Instituição & Região \\
\hline $\begin{array}{l}\text { N'UMBUNTU - Núcleo de Estudos, Pesquisa } \\
\text { e Extensão em Relações Étnico-Raciais, Movimentos Sociais } \\
\text { e Educação }\end{array}$ & $\begin{array}{l}\text { Universidade Federal do Sul e sudeste do } \\
\text { Pará }\end{array}$ & \multirow{15}{*}{ Norte $=15$} \\
\hline $\begin{array}{l}\text { NEABI - Núcleo de Estudos Afro-brasileiros e Indígenas do } \\
\text { IFRO }\end{array}$ & $\begin{array}{l}\text { Instituto Federal de Educação, Ciência e } \\
\text { Tecnologia de Rondônia }\end{array}$ & \\
\hline $\begin{array}{l}\text { GERA - Núcleo de Estudos e pesquisas sobre Formação de } \\
\text { Professores e Relações Étnico-Raciais }\end{array}$ & Universidade Federal do Pará & \\
\hline NEAB - Núcleo de Estudos Afro-brasileiros & Instituto Federal do Acre & \\
\hline NEAB/UNIFAP - Núcleo de Estudos Afro-Brasileiro & Universidade Federal do Amapá & \\
\hline NEABI - Núcleo de Estudos Afro-brasileiros e Indígenas & $\begin{array}{l}\text { Instituto Federal de Educação, Ciência e } \\
\text { Tecnologia do Pará, Campus Belém }\end{array}$ & \\
\hline NEAB - Núcleo de Estudos Afro-Brasileiros & Universidade Federal do Tocantins & \\
\hline $\begin{array}{l}\text { NEABI - Núcleo de Estudos e Pesquisas Afro-brasileiras e } \\
\text { Indígenas }\end{array}$ & Universidade Federal do Amazonas & \\
\hline NEAB - Núcleo de Estudos Afro-Brasileiros & Universidade do Estado do Amazonas & \\
\hline $\begin{array}{l}\text { NEABI - Núcleo de Estudos Afro-brasileiros } \\
\text { e Indígenas }\end{array}$ & $\begin{array}{l}\text { Instituto Federal de Educação, Ciência e } \\
\text { Tecnologia de Roraima - IFRR/Campus } \\
\text { Boa Vista Centro }\end{array}$ & \\
\hline $\begin{array}{l}\text { GEABI - Grupo de Estudos Afro-Brasileiros e Indígenas - } \\
\text { UFPA }\end{array}$ & Universidade Federal do Pará & \\
\hline Grupo de Estudos Afro amazônico - GEAM & $\begin{array}{l}\text { Universidade Federal do Pará } \\
\text { Campus de Belém }\end{array}$ & \\
\hline Núcleo de Estudos Afro-brasileiros & Universidade Estadual do Pará & \\
\hline $\begin{array}{l}\text { Grupo de Estudo e Pesquisa em Gênero, Juventude e } \\
\text { Etnicidade/Ubuntu }\end{array}$ & IFTO - Instituto Federal do Tocantins & \\
\hline Núcleo de Estudos Afro-Brasileiros e Indígenas & Universidade Federal do Acre - UFAC & \\
\hline $\begin{array}{l}\text { NEABI - Núcleo de Estudos e Pesquisas Afro-brasileiros e } \\
\text { Indígenas }\end{array}$ & Universidade Federal da Paraíba & \\
\hline NEAB - Núcleo de Estudos Afro-Brasileiros & Universidade Federal de Alagoas & \\
\hline $\begin{array}{l}\text { NEABI - Núcleo de Estudos Afro-brasileiros e Indio } \\
\text { descendentes }\end{array}$ & Instituto Federal do Maranhão & \\
\hline NEAB - Núcleo de Estudos Afro-Brasileiros & Universidade Federal do Maranhão & \\
\hline NEAB - Núcleo de Estudos Afro-Brasileiros & Universidade Federal do Sul da Bahia & \\
\hline $\begin{array}{l}\text { CEPAIA - Centro de Estudos dos Povos Afro-Indio- } \\
\text { Americano }\end{array}$ & Universidade do Estado da Bahia & \\
\hline $\begin{array}{l}\text { IFARADÁ - Núcleo de Pesquisa sobre Africanidades e } \\
\text { Afrodescendência }\end{array}$ & Universidade Federal do Piauí & \\
\hline NEAB - Núcleo de Estudos Afro-Brasileiros & Universidade Federal de Pernambuco & \\
\hline Núcleo de Estudos Africanos e Indígenas & Universidade Estadual da Região & \\
\hline
\end{tabular}




\begin{tabular}{|c|c|c|}
\hline & Tocantina do Maranhão & \multirow{24}{*}{ Nordeste $=32$} \\
\hline NEAB - Núcleo de Estudos Afro-brasileiros & $\begin{array}{l}\text { Universidade Federal Rural de } \\
\text { Pernambuco }\end{array}$ & \\
\hline NACE - Núcleo das Africanidades Cearenses & Universidade Federal do Ceará & \\
\hline ODEERE - Órgão de Educação das Relações Étnicas & $\begin{array}{l}\text { Universidade Estadual do Sudoeste da } \\
\text { Bahia - UESB }\end{array}$ & \\
\hline $\begin{array}{l}\text { NEABI - Núcleo de Estudos Afro Brasileiro e Indígenas - } \\
\text { Guarabira }\end{array}$ & $\begin{array}{l}\text { Instituição: Universidade Estadual da } \\
\text { Paraíba }\end{array}$ & \\
\hline $\begin{array}{l}\text { Coletivo Angela Davis - Grupo de Pesquisa em Gênero, Raça } \\
\text { e Subalternidade }\end{array}$ & $\begin{array}{l}\text { Universidade Federal do Recôncavo da } \\
\text { Bahia - Campus Cachoeira }\end{array}$ & \\
\hline NEAB & $\begin{array}{l}\text { Universidade de Integração Internacional } \\
\text { da Lusofonia Afro-brasileira - UNILAB } \\
\text { Campus de Liberdade }\end{array}$ & \\
\hline $\begin{array}{l}\text { Laboratório de Estudos e Pesquisas em Afro brasilidades, } \\
\text { Gênero e Família - NUAFRO }\end{array}$ & Universidade Estadual do Ceará & \\
\hline Núcleo de Estudos Afro-brasileiros e Indígenas & Universidade Federal de Sergipe & \\
\hline Núcleo de Estudos Afro Brasileiros e Indígenas & $\begin{array}{l}\text { Instituto Federal de Educação Ciência } \\
\text { Tecnologia do Sertão Pernambucano - } \\
\text { Campus Salgueiro }\end{array}$ & \\
\hline NEABI IF Baiano Campus Valença & Instituto Federal Baiano Campus Valença & \\
\hline NEABI IF Baiano - Campus Guanambi & IF Baiano Campus Guanambi & \\
\hline NEABI IF Baiano - Campus Itapetinga & IF Baiano Campus Itapetinga & \\
\hline $\begin{array}{l}\text { EtniCidades grupo de estudos étnicos e raciais em } \\
\text { arquitetura e urbanismo }\end{array}$ & $\begin{array}{l}\text { Faculdade de Arquitetura da Universidade } \\
\text { Federal da Bahia }\end{array}$ & \\
\hline Núcleo de Estudos Afro-brasileiros e Indígenas & $\begin{array}{l}\text { Instituto Federal de Educação, Ciência e } \\
\text { Tecnologia do Pará }\end{array}$ & \\
\hline $\begin{array}{l}\text { Laboratório de Educação das Relações Étnico-Raciais - } \\
\text { LabERER }\end{array}$ & Universidade Federal de Pernambuco & \\
\hline NÚCLEO DE ESTUDOS E PESQUISAS AFRO - NEPA & UESPI - Universidade Estadual do Piauí & \\
\hline Núcleo de Estudos Afro-brasileiro e Indígena & $\begin{array}{l}\text { Universidade do Estado da Bahia (UNEB) - } \\
\text { Campus XVIII }\end{array}$ & \\
\hline Núcleos de Estudos Afro-brasileiros e Indígenas & $\begin{array}{l}\text { Instituto Federal de Educação, Ciência e } \\
\text { Tecnologia - Ceará Campus - Juazeiro do } \\
\text { Norte }\end{array}$ & \\
\hline Núcleo de Estudos Afro-brasileiros e Indígenas (NEABI) & $\begin{array}{l}\text { Instituto Federal do Ceará - IFCE Campus } \\
\text { Jaguaribe }\end{array}$ & \\
\hline $\begin{array}{l}\text { AFROUNEB - Núcleo Interdisciplinar de Estudos Africanos e } \\
\text { Afro-Brasileiros }\end{array}$ & Universidade do Estado da Bahia - UNEB & \\
\hline Núcleo de Estudos Afro-brasileiros e Indígenas & $\begin{array}{l}\text { IFPI - Instituto Federal do Piauí - Campus } \\
\text { Cocal }\end{array}$ & \\
\hline NÚCLEO DE ESTUDOS AFRO-BRASILEIROS & $\begin{array}{l}\text { Universidade do Estado do Rio Grande do } \\
\text { Norte-UERN }\end{array}$ & \\
\hline NEABI-CAMPUS FORTALEZA & $\begin{array}{l}\text { Instituto Federal Ceará | Campus } \\
\text { Fortaleza }\end{array}$ & \\
\hline $\begin{array}{l}\text { NEGRA - Núcleo de Estudos sobre Educação, Gênero, Raça e } \\
\text { Alteridade }\end{array}$ & Universidade do Estado de Mato Grosso & \\
\hline NEAB - Núcleo de Estudos Afro-brasileiros & Universidade Federal da Grande Dourados & \\
\hline NEADI - Núcleo de Estudos Afrodescendente e Indígena & Universidade Federal de Goiás & \\
\hline NEAAD - Núcleo de Estudos Africanos e Afro-Diaspóricos & Universidade Estadual de Goiás - UEG & \\
\hline $\begin{array}{l}\text { NEGRA - Núcleo de Estudos de Gênero, Raça e } \\
\text { Africanidades }\end{array}$ & Instituto Federal de Goiás - IFG & \\
\hline $\begin{array}{l}\text { NUMDI - Núcleo de Estudos Afro-brasileiros, Indígena e de } \\
\text { Fronteira Maria Dimpina Lobo Duarte }\end{array}$ & $\begin{array}{l}\text { Instituto Federal de Educação, Ciência e } \\
\text { Tecnologia de Mato Grosso - } \\
\text { IFMT/Campus Fronteira Oeste/Pontes e } \\
\text { Lacerda }\end{array}$ & \\
\hline NEABI - Núcleo de Estudos Afro-brasileiros e Indígenas & Instituto Federal Goiano - IFGoiano & \\
\hline $\begin{array}{l}\text { GEPHERG - Grupo de Estudos e Pesquisas em Políticas } \\
\text { Públicas, História, Educação das Relações Raciais e Gênero }\end{array}$ & Universidade de Brasília - UnB & \\
\hline $\begin{array}{l}\text { CIATA-Laboratório de Pesquisas em Educação Química e } \\
\text { Inclusão LPEQI }\end{array}$ & Universidade Federal de Goiás & \\
\hline $\begin{array}{l}\text { PROAFRO - Núcleo de Estudos Africanos e Afro-brasileiros } \\
\text { do Programa de Estudos Africanos e Afro-brasileiros }\end{array}$ & Pontifícia Universidade Católica de Goiás & \\
\hline $\begin{array}{l}\text { CEPEGRE - Centro de Estudos, Pesquisa e Extensão em } \\
\text { Educação, Gênero, Raça e Etnia }\end{array}$ & $\begin{array}{l}\text { Universidade Estadual do Mato Grosso do } \\
\text { Sul }\end{array}$ & Centro Oeste $=20$ \\
\hline NEAB - Núcleo de Estudos Afro-brasileiros - UNB & Universidade de Brasília & \\
\hline $\begin{array}{l}\text { LaGENTE-Laboratório de estudos de gênero étnico-raciais e } \\
\text { especialidades }\end{array}$ & Universidade Federal de Goiás & \\
\hline $\begin{array}{l}\text { Núcleo de Estudos Afro Brasileiro e Indígena e de relações } \\
\text { de Gênero e Sexualidades (Neabí-Nuances) }\end{array}$ & $\begin{array}{l}\text { Instituto Federal de Goiás - Campus } \\
\text { Cidade de Goiás }\end{array}$ & \\
\hline
\end{tabular}




\begin{tabular}{|c|c|c|}
\hline $\begin{array}{l}\text { Núcleo de estudos e pesquisas sobre relações raciais e } \\
\text { educação }\end{array}$ & Universidade Federal do Mato Grosso & \\
\hline $\begin{array}{l}\text { NEABI - Núcleo de Estudos Afro-brasileiros e Indígenas - } \\
\text { IFGoiano }\end{array}$ & $\begin{array}{l}\text { Instituto Federal Goiano - IFGoiano - } \\
\text { Campus Posse }\end{array}$ & \\
\hline $\begin{array}{l}\text { Núcleo de Estudos Afro-brasileiros e Indígenas da } \\
\text { Universidade Federal de Mato Grosso do Sul }\end{array}$ & $\begin{array}{l}\text { Universidade Federal de Mato } \\
\text { Grosso do Sul }\end{array}$ & \\
\hline $\begin{array}{l}\text { Núcleo de Estudos Afro-brasileiros da Universidade Federal } \\
\text { de Tocantins }\end{array}$ & Universidade Federal de Tocantins & \\
\hline $\begin{array}{l}\text { NUMDI - Núcleo de Estudos Afro-brasileiros, Indígena e de } \\
\text { Fronteira Maria Dimpina Lobo Duarte }\end{array}$ & $\begin{array}{l}\text { Instituto Federal de Educação, Ciência e } \\
\text { Tecnologia de Mato Grosso - } \\
\text { IFMT/REITORIA }\end{array}$ & \\
\hline Núcleo de Estudos Afro-brasileiros - NEAB & Universidade Federal de Uberlândia & \\
\hline ONDJANGO - Núcleo de Estudos Afro-brasileiros & $\begin{array}{l}\text { Fundação de Apoio à Escola Técnica do } \\
\text { Estado do Rio de Janeiro - FAETEC / Escola } \\
\text { Técnica João Luiz do Nascimento }\end{array}$ & \multirow{28}{*}{ Sudeste $=36$} \\
\hline $\begin{array}{l}\text { NEABCP2 - Núcleo de Estudos Afro-Brasileiros do Colégio } \\
\text { Pedro II }\end{array}$ & Colégio Pedro II & \\
\hline $\begin{array}{l}\text { NEAB/UFJF - Núcleo de Estudos Afro-brasileiros da } \\
\text { Universidade Federal de Juiz de Fora }\end{array}$ & Universidade Federal de Juiz de Fora & \\
\hline $\begin{array}{l}\text { NEABI - Núcleo de Estudos Afro-Brasileiros e Indígenas - } \\
\text { UFOP }\end{array}$ & Universidade Federal de Ouro Preto & \\
\hline $\begin{array}{l}\text { LEAFRO - Laboratório de Estudos Afro-Brasileiros e } \\
\text { Indígenas }\end{array}$ & $\begin{array}{l}\text { UFRRJ - Universidade Federal Rural do Rio } \\
\text { de Janeiro }\end{array}$ & \\
\hline NEAB - Núcleo de Estudos Afro-brasileiros & $\begin{array}{l}\text { Centro Federal de Educação Tecnológica } \\
\text { Celso Suckow da Fonseca }\end{array}$ & \\
\hline $\begin{array}{l}\text { NEIAB - Núcleo de Estudos Indígena e Afro-brasileiro - } \\
\text { UNAERP }\end{array}$ & Universidade de Ribeirão Preto & \\
\hline NEABI - IF SUDESTE MG & $\begin{array}{l}\text { Instituto Federal de Educação, Ciência e } \\
\text { Tecnologia do Sudeste de Minas Gerais - } \\
\text { IF Sudeste MG }\end{array}$ & \\
\hline NEAB - Núcleo de Estudos Afro-brasileiros & $\begin{array}{l}\text { Centro Federal de Educação Tecnológica } \\
\text { Celso Suckow da Fonseca }\end{array}$ & \\
\hline $\begin{array}{l}\text { NEABI - Núcleo de Estudos Afro-Brasileiros e Indígenas - } \\
\text { UENF }\end{array}$ & $\begin{array}{l}\text { Universidade Estadual do Norte } \\
\text { Fluminense Darcy Ribeiro }\end{array}$ & \\
\hline NEAB - Núcleo de Estudos Afro-brasileiros - UNIFESP & Universidade Federal de São Paulo & \\
\hline $\begin{array}{l}\text { Programa de Educação sobre o negro na sociedade } \\
\text { brasileira }\end{array}$ & Universidade Federal Fluminense & \\
\hline NEAB - Núcleo de Estudos Afro-brasileiros NEAB/UFES & Universidade Federal do Espírito Santo & \\
\hline NEAB - Núcleo de Estudos Afro-brasileiros & Universidade do Estado do Rio de Janeiro & \\
\hline NEAB - Núcleo de Pesquisa e Estudos Afro-Brasileiros & $\begin{array}{l}\text { Centro Federal de Educação Tecnológica } \\
\text { de Minas Gerais }\end{array}$ & \\
\hline Programa de Ações Afirmativas da UFMG & Universidade do Estado de Minas Gerais & \\
\hline Programa Institucional de Ações Afirmativas UEMG & $\begin{array}{l}\text { Universidade do Estado de Minas Gerais - } \\
\text { Campus Ubá }\end{array}$ & \\
\hline $\begin{array}{l}\text { LICAFRO - Laboratório de Literaturas e Culturas Africanas e } \\
\text { da Diáspora Negra }\end{array}$ & $\begin{array}{l}\text { Universidade Federal Fluminense - } \\
\text { Campus Gragoata }\end{array}$ & \\
\hline NEABI-Cpar & $\begin{array}{l}\text { Instituto Federal do Rio de Janeiro } \\
\text { Campus Paracambi - IFRJ }\end{array}$ & \\
\hline $\begin{array}{l}\text { Nupe - Núcleo negro para pesquisa e extensão da Unesp - } \\
\text { GT de Rio Preto }\end{array}$ & $\begin{array}{l}\text { Universidade Estadual Paulista Júlio de } \\
\text { Mesquita Filho' - Campus São Jose do Rio } \\
\text { Preto }\end{array}$ & \\
\hline $\begin{array}{l}\text { Programa de Estudos e Debates dos Povos Africanos e Afro- } \\
\text { Americanos - PROAFRO }\end{array}$ & $\begin{array}{l}\text { Universidade do Estado do Rio de Janeiro } \\
\text { - Campus Maracanã - UERJ }\end{array}$ & \\
\hline NEAB NIEHLAFRO & $\begin{array}{l}\text { Universidade do Estado de Minas Gerais - } \\
\text { Campus Belo Horizonte }\end{array}$ & \\
\hline NEAB/IFMG/OP & $\begin{array}{l}\text { Instituto Federal de Educação, Ciência e } \\
\text { Tecnologia de Minas Gerais - Campus } \\
\text { Ouro Preto - IFMG }\end{array}$ & \\
\hline Neab Ufscar & Universidade Federal de São Carlos & \\
\hline $\begin{array}{l}\text { Núcleo de Estudos Afro-Brasileiros e Indígenas - NEABI } \\
\text { Campus Ituiutaba }\end{array}$ & $\begin{array}{l}\text { Instituto Federal de Educação, Ciência e } \\
\text { Tecnologia do Triângulo Mineiro - Campus } \\
\text { Ituiutaba - IFTM }\end{array}$ & \\
\hline Núcleo de Estudos Afro brasileiros e Indígenas do IFSP & $\begin{array}{l}\text { Instituto Federal de Educação Ciência e } \\
\text { Tecnologia do Estado de São Paulo }\end{array}$ & \\
\hline $\begin{array}{l}\text { Núcleo de Estudos Afro-brasileiro e Indígena do Campus Rio } \\
\text { de Janeiro }\end{array}$ & $\begin{array}{l}\text { Instituto Federal de Educação Ciência e } \\
\text { Tecnologia do Rio de Janeiro }\end{array}$ & \\
\hline NEABI Macaé & $\begin{array}{l}\text { Universidade Federal do Rio de Janeiro - } \\
\text { Campus Macaé e Instituto de Ciências da }\end{array}$ & \\
\hline
\end{tabular}




\begin{tabular}{|c|c|c|}
\hline & Sociedade de Macaé/UFF & \\
\hline Observatório da Diversidade & Instituto Federal de Minas Gerais - IFMG & \\
\hline $\begin{array}{l}\text { Núcleo de Estudos Afro-Brasileiros - Universidade Federal } \\
\text { Fluminense/Campus Rio das Ostras }\end{array}$ & Universidade Federal Fluminense & \\
\hline NEABI Pontal & Universidade Federal de Uberlândia & \\
\hline Núcleo de Estudos Africanos e Afro-brasileiros & Universidade Federal do $A B C$ & \\
\hline Núcleo de Estudos Afro-brasileiros Ayó - NEAB-AYÓ & Escola Municipal Clementino Fraga & \\
\hline Núcleo de Estudos Afro-brasileiros e Indígenas & Instituto Federal do Rio de Janeiro & \\
\hline $\begin{array}{l}\text { Núcleo de Estudos afro brasileiro e indígenas da } \\
\text { Universidade Federal dos Vales do Jequitinhonha e Mucuri }\end{array}$ & $\begin{array}{l}\text { Universidade Federal dos Vales do } \\
\text { Jequitinhonha e Mucuri- Minas Gerais }\end{array}$ & \\
\hline $\begin{array}{l}\text { Núcleo de Estudos Afro-Brasileiros e Indígenas (Neabi) do } \\
\text { Campus Colatina }\end{array}$ & $\begin{array}{l}\text { Instituto Federal do Espírito Santo - } \\
\text { Campus Colatina }\end{array}$ & \\
\hline NEABI - Núcleo de Estudos Afro-Brasileiros e Indígenas & $\begin{array}{l}\text { Instituto Federal de Santa Catarina - } \\
\text { IFSC/Campus Gaspar }\end{array}$ & \multirow{32}{*}{ Sul $=36$} \\
\hline NEAB - Núcleo de Estudos Afro-brasileiros & $\begin{array}{l}\text { Universidade do Extremo Sul Catarinense } \\
\text { - UNESC }\end{array}$ & \\
\hline Núcleo de Relações Étnico Raciais, de Gênero e Sexualidade & Universidade Estadual de Ponta Grossa & \\
\hline Grupo de Estudos Etno-Culturais & $\begin{array}{l}\text { Universidade Estadual do Oeste do Paraná } \\
\text { - Unioeste }\end{array}$ & \\
\hline NEAA - Núcleo de Estudos Ameríndios e Africanos & $\begin{array}{l}\text { Universidade Estadual do Centro-Oeste - } \\
\text { UNICENTRO }\end{array}$ & \\
\hline NEAB - Núcleo de Estudos Afro-brasileiros & Universidade Federal do Paraná & \\
\hline NEAB - Núcleo de Estudos Afro-brasileiros & Universidade Regional de Blumenau & \\
\hline $\begin{array}{l}\text { NEABI - Núcleo de Estudos em Cultura Afro-Brasileira e } \\
\text { Indígena }\end{array}$ & $\begin{array}{l}\text { Pontifícia Universidade Católica do Rio } \\
\text { Grande do Sul-PUC/RS }\end{array}$ & \\
\hline NUVIC - Grupo de Estudos sobre Violências & Universidade Federal de Santa Catarina & \\
\hline NEAB - Núcleo de Estudos Afro-brasileiros & Universidade do Estado de Santa Catarina & \\
\hline NEAB - Núcleo de Estudos Afro-brasileiros & $\begin{array}{l}\text { Universidade Federal do Pampa - } \\
\text { Unipampa }\end{array}$ & \\
\hline NEIA - Núcleo de Estudos Interdisciplinares Afro-brasileiros & Universidade Estadual de Maringá & \\
\hline NEABI - Núcleo de Estudos Afro-Brasileiros e Indígenas & Universidade Luterana do Brasil & \\
\hline ALTERITAS: Diferença, Arte e Educação & $\begin{array}{l}\text { Universidade Federal de Santa Catarina - } \\
\text { UFSC }\end{array}$ & \\
\hline NEABI - Núcleo de Estudos Afro-brasileiros e Indígenas & Universidade do Vale do Rio dos Sinos & \\
\hline $\begin{array}{l}\text { Núcleo de Estudos Afro-brasileiros e Indígenas - Negro e } \\
\text { Educação }\end{array}$ & Universidade do Planalto Catarinense & \\
\hline Núcleo de Estudos Afro-Brasileiros e Indígenas FURG & Universidade Federal do Rio Grande & \\
\hline NEABI - Núcleo de Estudos Afro-brasileiros e Indígenas & $\begin{array}{c}\text { Instituto Federal de Educação Ciência e } \\
\text { Tecnologia do Rio Grande do Sul - IFRS/ } \\
\text { Campus Porto Alegre }\end{array}$ & \\
\hline $\begin{array}{l}\text { Instituto Federal de Educação, Ciência e Tecnologia do Rio } \\
\text { Grande do Sul IFRS/ /Campus Sertão }\end{array}$ & $\begin{array}{l}\text { Instituto Federal de Educação, Ciência e } \\
\text { Tecnologia do Rio Grande do Sul IFRS }\end{array}$ & \\
\hline NEABI - Núcleo de Estudos Afro-brasileiros e Indígenas & $\begin{array}{l}\text { Instituto Federal de Educação, Ciência e } \\
\text { Tecnologia do Rio Grande do Sul - } \\
\text { IFRS/Campus Bento Gonçalves }\end{array}$ & \\
\hline $\begin{array}{l}\text { NAAIA - Núcleo de Ações Afirmativas do IFRS Campus } \\
\text { Alvorada }\end{array}$ & $\begin{array}{l}\text { Instituição: Instituto Federal de Educação } \\
\text { Ciência e Tecnologia do Rio Grande do Sul } \\
\text { - IFRS/Campus Alvorada }\end{array}$ & \\
\hline NEABI - Núcleo de Estudos Afro-brasileiros e Indígenas & $\begin{array}{l}\text { Instituto Federal de Educação Ciência e } \\
\text { Tecnologia do Rio Grande do Sul - } \\
\text { IFRS/Campus Canoas }\end{array}$ & \\
\hline NEABI - Campus Feliz & Instituto Federal do Rio Grande do Sul & \\
\hline $\begin{array}{l}\text { Núcleo de Estudos Afro-Brasileiros e Indígenas - UFFS } \\
\text { Campus Chapecó }\end{array}$ & Universidade Federal da Fronteira do Sul & \\
\hline NEAB: Núcleo de Estudos Afro-Brasileiros UNIVILLE & Universidade da Região de Joinville & \\
\hline NEAB & $\begin{array}{l}\text { Universidade Federal do Pampa - } \\
\text { Unipampa - Campus São Borja }\end{array}$ & \\
\hline NEAB & $\begin{array}{l}\text { Universidade Federal do Pampa - } \\
\text { Unipampa - Campus Jaguarão }\end{array}$ & \\
\hline Núcleo de Estudos Afro-Brasileiros e Indígenas - NEABI & $\begin{array}{l}\text { Universidade Federal do Pampa - } \\
\text { Unipampa - Campus Itaqui }\end{array}$ & \\
\hline NEAB Estácio SC & $\begin{array}{l}\text { Universidade Estácio de Sá - Santa } \\
\text { Catarina }\end{array}$ & \\
\hline $\begin{array}{l}\text { NEALA: Núcleo de Estudos Afro Latino Americanos e } \\
\text { Caribenhos }\end{array}$ & $\begin{array}{l}\text { UNILA - Universidade Federal da } \\
\text { Integração Latino Americana }\end{array}$ & \\
\hline NEABI/UFFS-ERE & $\begin{array}{l}\text { Universidade Federal da Fronteira Sul, } \\
\text { Campus Erechim }\end{array}$ & \\
\hline $\begin{array}{l}\text { Laboratório de Cultura e Estudos Afro-Brasileiros e } \\
\text { Africanos - LEAFRO }\end{array}$ & $\begin{array}{l}\text { Universidade Estadual de Londrina - } \\
\text { Campus Londrina }\end{array}$ & \\
\hline
\end{tabular}




\begin{tabular}{|l|l|}
\hline Núcleo de Estudos Afro-Brasileiros da UEL - NEAB/UEL & $\begin{array}{l}\text { Universidade Estadual de Londrina - } \\
\text { Campus Londrina }\end{array}$ \\
\hline Núcleo de Estudos Afro-Brasileiros, Indígenas e Africanos & $\begin{array}{l}\text { Universidade Federal do Rio Grande do } \\
\text { Sul }\end{array}$ \\
\hline $\begin{array}{l}\text { Núcleo de Estudos Afro-Brasileiros e Indígenas do Instituto } \\
\text { Federal do Paraná }\end{array}$ & $\mathbf{1}$ \\
\hline $\begin{array}{l}\text { Núcleo de Estudos Afro-brasileiros e Indígenas da } \\
\text { UNIPAMPA - NEABI MOCINHA }\end{array}$ & $\begin{array}{l}\mathbf{2} \\
\text { Pampa }\end{array}$
\end{tabular}

Fonte: retirado do site : https://www.abpn.org.br/consorcio-de-neabs e organizados pelos autores

Quadro 3. Museus Afro-Brasileiros

\begin{tabular}{|c|c|c|c|}
\hline Museu & Cidade & $\begin{array}{l}\text { Público ou } \\
\text { privado }\end{array}$ & Região \\
\hline Cafua das Mercês (Museu do Negro) & São Luís, MA & Público & \multirow{18}{*}{ Nordeste } \\
\hline Museu da Abolição & Recife-PE & Público & \\
\hline Museu de Artes Afro-Brasil Rolando Toro & Recife, $\mathrm{PE}$ & Privado & \\
\hline Museu Senzala Negro Liberto & Redenção, CE & Privado & \\
\hline Museu Afro-brasileiro de Sergipe & Laranjeiras, SE & Público & \\
\hline Museu Casa do Benin & Salvado-BA & Público & \\
\hline Museu Último Quilombo & Boa Vista do Tupim, BA & Público & \\
\hline Museu Afro Cultural Oyá Ní & Alagoinhas, BA & Privado & \\
\hline Memorial Lajoumim - Terreiro Pilão de Prata & Salvador, BA & Privado & \\
\hline $\begin{array}{l}\text { Museu Digital da Memória Afro-Brasileira e } \\
\text { Africana (Virtual) }\end{array}$ & Salvador, BA & Público & \\
\hline Museu Ilê Ohun Lailai & Salvador, BA & Privado & \\
\hline Museu Comunitário Mãe Mirinha de Portão & Lauro de Freitas, BA & Privado & \\
\hline Memorial Kisimbiê - Águas do Saber & Salvador, BA & Privado & \\
\hline Museu Nacional da Cultura Afro-brasileira & Salvador, BA & Público & \\
\hline Museu Afro Omon Ajagunan & Lauro de Freitas, BA & Privado & \\
\hline $\begin{array}{l}\text { Memorial Mãe Menininha do Gantois/Associação } \\
\text { de São Jorge Ebé Oxossi }\end{array}$ & Salvador, BA & Privado & \\
\hline $\begin{array}{l}\text { Museu Afro-Brasileiro da Universidade Federal da } \\
\text { Bahia }\end{array}$ & Salvador, BA & Público & \\
\hline Parque Memorial Quilombo dos Palmares & União dos Palmares, AL & Público & \\
\hline $\begin{array}{l}\text { Museu Capixaba do Negro "Verônica da Pas" - } \\
\text { Mucane }\end{array}$ & Vitória, ES & Público & \multirow{14}{*}{ Sudeste } \\
\hline Museu dos Quilombos e Favelas Urbanos & Belo Horizonte, MG & Privado & \\
\hline Museu do Escravo (Belo Vale) & Belo Vale, MG & Público & \\
\hline Centro de Referência da Cultura Negra & Araxá, MG & Público & \\
\hline Instituto de Pesquisas Afro Cultural Odé Gbomi & Nova Iguaçu, RJ & Privado & \\
\hline Museu de Favela & Rio de Janeiro, RJ & Privado & \\
\hline $\begin{array}{l}\text { Memorial Afro-Valenciano Padre João José da } \\
\text { Rocha }\end{array}$ & Valença, RJ & Privado & \\
\hline Museu do Negro & Rio de Janeiro, RJ & Privado & \\
\hline Museu do Escravo & Barra do Piraí, RJ & Privado & \\
\hline Museu Afro Brasil & São Paulo-SP & Público & \\
\hline Museu do Negro de Campinas & Campinas, SP & Privado & \\
\hline Museu da Cultura Africana e Negritude Brasileira & Amparo, SP & Privado & \\
\hline Òsun Ìya Oke Ile Afro-Brasileiro Ode Lorecy & Embu das Artes, SP & Privado & \\
\hline $\begin{array}{l}\text { Casa da Cultura Afro-Brasileira - Memorial ao } \\
\text { Escravizado }\end{array}$ & São Vicente, SP & $\begin{array}{l}\text { Museu } \\
\text { Público }\end{array}$ & \\
\hline Casa da Cultura Quilombo & Quilombo, SC & Público & \multirow{4}{*}{ Sul } \\
\hline Casa dos Açores - Museu Etnográfico & Biguaçu, SC & Público & \\
\hline Museu Treze de Maio & Santa Maria, RS & Privado & \\
\hline Museu Paroquial Padre Osmar Possamai & São Marcos, RS & Privado & \\
\hline
\end{tabular}

Fonte: informações retiradas do site: http://museus.cultura.gov.br/ e elaborado pelo autores 
O mapeamento serviu para evidenciar a quantidade expressiva de unidades de informações que preservam a memória e história das populações de origem africana via coleções formadas por objetos e fontes bibliográficas que, por conseguinte, servem para construir novos conhecimentos. A unidades de informações privadas, as relacionadas aos terreiros de candomblé, são reconhecidas como patrimônio material e imaterial devido a relevância histórica e cultural.

Quando falamos de patrimônio, os aspectos materiais sempre aparecerão conexos com as questões imateriais (ALONSO, 2016). O físico e o simbólico se apresentam simultaneamente, o objeto só se torna patrimônio porque tem uma carga simbólica que lhe foi atribuída por uma pessoa, um grupo, uma sociedade.

A cultura material é fundamental para manter e reproduzir práticas africanas, sejam elas de sobrevivência, resistência, domésticas, religiosas, culturais; os objetos possuem uma utilidade e um simbolismo, fazem parte da identidade cultural afro-brasileira (ROSA, 2010).

Em um país com histórico de quase quatro séculos de escravidão e que tenta apagar ou minimizar os impactos dessa realidade para a população negra, se configura em um país estruturalmente racista. Conforme argumenta Mendes (2014, p. 2), historicamente é negado ao negro o papel de sujeito histórico, com a subsequente desvalorização de tudo que se vincula a essa população. É nesse sentido que a existência e disseminação dessas coleções é uma das maneiras de valorizar as heranças e reconhecer a importância e o protagonismos a partir do olhar da população de origem africana, de quem deixou registrado via material e objetos os acontecimentos histórico, cultural e social.

\section{Conclusão}

A proposta deste trabalho foi traçar um panorama da história do colecionismo, sua relação com a memória social de diversos povos, em especial dos afro-brasileiros, refletindo sobre o porquê das coleções existirem no mundo e ainda como elas podem contribuir para o desenvolvimento social, cultural e educacional, conforme prevê a Lei Federal 10.639/03 ${ }^{2}$ A simples existência de coleções pode nos dizer muita coisa, cultural e historicamente falando. Porém, somente o planejamento estratégico e integrado entre diferentes instituições, públicas e privadas, para disponibilização, preservação, disseminação e uso dessas coleções é que pode trazer contributos para a sociedade.

As coleções, enquanto fontes de informações, signos materiais da memória - quando

\footnotetext{
${ }^{2}$ Altera a Lei n. 9.394, de 20 de dezembro de 1996, que estabelece as diretrizes e bases da educação nacional, para incluir no currículo oficial da Rede de Ensino a obrigatoriedade da temática "História e Cultura Afro-Brasileira", e dá outras providências (BRASIL, 2003)
} 
vinculadas à explicações sobre o contexto histórico, social, político, econômico no qual foram formadas, ou do qual advém seus objetos, representam oportunidades de aprendizado e reconhecimento dos povos historicamente marginalizado. Entender que essas coleções podem e devem ser utilizadas como um dos recursos para combater o preconceito, o racismo e a intolerância, que se encontram embasadas na ignorância e na distorção de fatos.

As coleções (ou algumas delas) podem ser um patrimônio, podem ser mantidas como uma forma de manter o passado vivo em nossas memórias, para que possamos aprender com ele. A transmissão da história e significados atrelados aos objetos e demais documentos de uma coleção é, em tais casos, mais importantes do que a própria existência da coleção.

A UNESCO, através do ICOM (Conselho Internacional de Museus - em inglês: International Council of Museums) orienta que a preservação precisa estar aliada à iniciativas de uso e educação através dessas coleções. Portanto, a simples existência dessas coleções não basta, isso se constitui apenas o primeiro passo. É imprescindível que essas coleções cheguem até a população, que sejam adotadas nas práticas de ensino, estejam integradas aos currículos básicos das escolas e universidades. Que o seu conhecimento e acesso não dependa apenas de iniciativas e interesses individuais, mas faça parte de políticas públicas.

Os estudos sobre o mapeamento das possíveis coleções (objetos e bibliografias) nas instituições públicas privadas, que totalizam 181, tais como Centros de Estudos Africanos, Núcleo de Estudos Afro-Brasileiros e Museus, não param por aqui, para as futuras pesquisas é importante selecionar um museu privado para identificação dos objetos, reconstituição de sua origem e os significados tanto para o museu quanto para a comunidade ao seu redor.

\section{Referências}

ANTONACCI, M. A. Memórias ancoradas em corpos negros. São Paulo: Educ, 2015.

ARQUIVO NACIONAL. Dicionário brasileiro de terminologia arquivística. Rio de Janeiro: Arquivo Nacional, 2005.

AZEVEDO, R. L. T. O "Espaço Cassiano Nunes" e o desenvolvimento de coleções da Biblioteca Central da Universidade de Brasília: a aplicação da política de seleção ao colecionismo. Monografia (Graduação em Biblioteconomia). Brasília: UNB, 2015.

BAUDRILLARD, J. O sistema dos objetos. 4. ed. São Paulo: Perspectiva, 2004.

BERNARDINO-COSTA, J.; SANTOS, S. A.; SILVÉRIO, V. R. Relações raciais em perspectiva. Sociedade e Cultura, Goiânia, v. 12, n. 2, p. 215-222, jul./dez. 2009.

BIBLIOTECA NACIONAL (Rio de Janeiro). Para uma História do Negro no Brasil. Rio de Janeiro: Biblioteca Nacional, $1988.65 \quad$ p. 65 Disponível em: http://objdigital.bn.br/acervo digital/div iconografia/icon1104317/icon1104317.pdf Acesso em: 5 set. 2019. 
BRASIL. MINISTÉRIO DA EDUCAÇÃO. Diretrizes Curriculares Nacionais para a Educação das Relações Étnico-Racial e para o Ensino de História e Cultura Afro-brasileira e Africana. Brasília, DF: MEC, SEPPIR, SECAD, INEP, 2004.

BUENO, F. da S. Grande Dicionário Etimológico-Prosódico da Língua Portuguesa. São Paulo: Editora Brasília, 1974.

CAMPOS, A. C. B. et al. Narrativas e Contos Africanos: o resgate da tradição oral a partir das narrativas dos Griots. In: GERLIN, M. N. M. (Org.). Competência em Informação e narrativa numa sociedade conectada por redes. v. 2. Brasília: Faculdade de Ciência da Informação, 2018. p. 222-237.

CARDOSO, P. de J. F.; RASCKE, K. L. Lei Federal 10.639/03, discussão de conceitos: multiculturalismo, diversidade, ações afirmativas, racismo, preconceito, afrodescendente, negro, entre outros. In: CARDOSO, P. de J. F.; RASCKE, K. L. (Org.). Formação de professores: promoção e difusão de conteúdos sobre história e cultura afro-brasileira e africana. Florianópolis: DIOESC, 2014.

DOMINGUES, P. J. Movimento da negritude: uma breve reconstrução histórica. África: Revista do Centro de Estudos Africanos, São Paulo, v. 24-25-26, p. 193-210, 2009.

DOMINGUES, P. J. O mito da democracia racial e a mestiçagem no Brasil (1889-1930). Diálogos Latino-americanos, Aarhus, n. 10, p. 116-131, 2005.

FARINA, M. C.; TOLEDO, G. L.; CORRÊA, G. B. F. Colecionismo: uma perspectiva abrangente sobre o comportamento do consumidor. In: SEMEAD - Seminários em Administração FEA-USP, 9, São Paulo, 2006. Anais... 2006. São Paulo, 2006.

FELIPE, D. A.; TERUYA, T. K. Ensino da História e Cultura Africana em salas de aula brasileira. In: SEMINÁRIO DE PESQUISA DO PPE. Maringá, 23 e 28 de abril de 2010. Anais... Maringá: Universidade Estadual de Maringá, 2010.

FONSECA, P. Considerações de um colecionador. Episteme, Porto Alegre, v. 1, n. 20, suplemento especial, p. 181- 184, jan./jun. 2005.

FOUCAULT, M. A ordem do discurso. São Paulo: Edições Loyola. 1973.

HOMULOS, P. Museums to libraries: a family of collecting institutions. Art Libraries Journal, v. 15, n. 1, p. 11-13, 1990.

HUYSSEN, A. Seduzidos pela memória. Rio de Janeiro: Aeroplano, 2000.

MENDES, C. J. O colecionismo de objetos africanos e afrodescendentes no atlântico português - 1822-1960. In: CONGRESSO INTERNACIONAL DE HISTÓRIA, 4, 2014, Jataí. Anais... Universidade Federal de Goiás, 2014.

MENEGAT, R. A epistemologia e o espírito do colecionismo. Episteme. Porto Alegre, n. 20, p. 512, jan./jun., 2005. 
MUNANGA, K. Uma Abordagem Conceitual das Noções de Raça, Racismo, Identidade e Etnia. [Artigo on-line]. In: SEMINÁRIO NACIONAL RELAÇÕES RACIAIS E EDUCAÇÃO-PENESB-RJ, 3., Rio de Janeiro: Palestra proferida em 5 nov. 2003, p. 1-17, 2003.

MORTARI, C.; VIEIRA, F. A. O Brasil dos séculos XVI a XIX: populações de origem africana, cativeiro, identidades, solidariedades, religiosidade e resistências. In: CARDOSO, P. de J. F.; RASCKE, K. L. (Org.). Formação de professores: promoção e difusão de conteúdos sobre história e cultura afro-brasileira e africana. Florianópolis: DIOESC, 2014.

NORA, P. Entre memória e história: a problemática dos lugares. Projeto História. São Paulo: PUC-SP. N 10, p. 12. 1993.

OGBECHIE, S. O. O Museu Africano Pós-Colonial na Era da Informática Cultural. biblioteca.pinacoteca, 2014.2 Disponível em: http://biblioteca.pinacoteca.org.br:9090/publicacoes/index.php/sim/article/view/71 Acesso em: 12 nov. 2019.

OLIVEIRA, D. F. H.; HOLANDA, A. F.; MACIEL, Josemar de Campos. Coleções e colecionadores: compreendendo o significado de colecionar. Revista NUFEN, Belém, v. 8, n. 1, p. 31-54, 2016.

PEDRÃO, G. B.; BIZELLO, M. L. As coleções como patrimônio: um meio para a preservação da história e da memória. In: SEMINÁRIO EM CIÊNCIA DA INFORMAÇÃO, 6, Londrina, Anais.... Londrina, 2016. p. 829-840.

POMIAN, K. Coleção. In: GIL, F. (Org.). Enciclopédia Einaudi. Volume 1 Memória-História. Porto: Imprensa Nacional: Casa da Moeda, 1984. p. 51-86.

RENAULT, L. V. O ato colecionador. (Dissertação de Doutorado), UFMG, Belo Horizonte, 2015. Disponivel em: http://hdl.handle.net/1843/BUBD-9ZLPDN

ROSA, E. J. Identidade Afro-brasileira: Um diálogo entre Memória e Cultura material. Revista Memória em Rede, Pelotas, v. 2, n. 3,. p. 59-71, ago./nov., 2010.

SERRANO, C.; WALDMAN, M. Memória d’África: A temática africana em sala de aula. São Paulo: Cortez, 2007.

SILVA, F. A. do N. da. Africanidade e valorização da cultura negra na formação da cultura brasileira. 2013. 55 f. Monografia (Especialização em Histórias e Culturas Afro-Brasileira, Indígena e Africana) - Universidade da Integração Internacional da Lusofonia Afro-brasileira, Redenção, CE, 2014.

VALIS, N. El coleccionismo: el rescate de las cosas y lo humano. Letras de Hoje, Porto Alegre, v. 48, n. 4, p. 559-568, out./dez., 2013. 